\title{
Time to make friends of chemists
}

The contemporary chemistry journals deserve to be more widely read, not just for what chemists have to say about the natural world, but for the daring of the empirical generalizations in which chemists seem to specialize.

Chemists are much misunderstood, at least in part because of their publishing practices. Potential well-wishers have been kept from recognizing the value of what they do by the way chemistry journals are spattered with articles described as "Part XIII" in a continuing study of some class of compounds, texts are filled with formal characterizations of whichever compounds may have been synthesized in the course of a study, much as taxonomists record the classification of new species.

Yet ask a chemist what he or she is about, and you will be given a different tale. First, of course, you will be told about the useful arts, the processes for making materials as different as polypropylene and Semtex (the 'plastic' explosive used for blowing up civilian aircraft). At least to outsiders, there now seem no limits to what people can synthesize. They are even prepared to do it with one hand tied behind their back: a recent issue of Journal of the American Chemical Society contains an account by Nakatsuka et al. $(112,5583 ; 1990)$ of a synthesis of the immunosuppressant FK506 in which two specific carbon atoms were substituted by ${ }^{13} \mathrm{C}$, and in which the authors used $\alpha$-bromoacetic acid in their synthesis scheme because it is readily (and cheaply) available as a material in which both carbons are enriched in the heavy isotope.

Yet chemists' most engaging habit is their confidence that chemistry is an explanation of virtually everything. Physicists may break their heads over the existence of the top quark, or whether axions can supply the missing mass of the Universe, but chemists will not shrink from explaining why Teflon has virtually no friction or why zeolites function well as catalysts for cracking hydrocarbons.

Outsiders should not disparage such impatient ambition. Chemists are masters at elevating empirical generalizations into natural laws. Is there not, for example, Trouton's rule that the latent heat of vaporization of a liquid in calories is roughly 22 times the boiling point (in degrees kelvin)?

For half a century after Mendeleev, the periodic table of the elements must have seemed to most of them to be a preferred substitute for Aristotle's elementary bundle of earth, air, fire and water. The arrival of the quantum theory and, in particular, of Pauli's exclusion principle (which explains the periodic table except for the three-quarters of the elements whose atomic number is greater than that of iron) did not seem to chemists of the time to be an undercutting of their theory, but rather a confirmation of what they had been saying all along. If only somebody, in 1917, had had the wit to announce that Einstein's general theory had confirmed that newtonian mechanics is essentially correct, physicists would have avoided much subsequent trouble.

Generalizations based on observations have at least the virtue that they are generalizations of the kind ordinary people reach in ordinary life. Could that have been, in past decades, one of the reasons why chemists were able to attract large numbers of others like themselves to follow their own pursuits? More recently, faced with falling enrolments in student courses, chemists and their professional organizations have been tempted to emphasize the useful arts. Might they not find it at least as effective to describe chemistry as a way of reaching accessible, if sometimes empirical, explanations of the natural world?

Here is another illustration, from the same issue of J. Am. chem. Soc. as the synthesis of FK506 and the account (see last week's Nature 347, 13; 1990) by Hunter and Sanders of how the interaction between conjugated electron systems can be simplified.

Take, for example, pyridine, the analogue of the conjugated six-carbon benzene molecule in which one of the carbon atoms is replaced by a nitrogen, with the result that the molecule contains only five hydrogen atoms (one for each carbon). Should this not be essentially the same as benzene? Not exactly. Pyridine resembles benzene in the old Kekulé picture of conjugated systems in which the successive atoms in the six-membered ring are joined by alternating single and double bonds, but then there are two electrons left over (nitrogen has five electrons in the second shell, but only three are used up in making bonds), usually termed a 'lone pair'. Hand-waving over many years has been taken to suggest that this is why pyridine is more reactive chemically than benzene. More to the point, calculations show that the ground-state of the molecule is indeed planar, like that of benzene.

But what about the excited states of pyridine? The analogy with benzene is no longer reliable, for the two spare electrons on the nitrogen atom are natural candidates for excitation. Apparently the spectroscopy of pyridine has been a head- ache, sorted out only in the past few years by the spectral observation of deuterated pyridine in crystals of deuterated benzene. The first excited state is a triplet state, and the observations show the molecule to be boat-shaped (with the nitrogen atom lifted from the plane by about $35^{\circ}$, the diametrically opposite carbon atom by about a third as much in the same direction).

Why should this be? W. J. Buma, E. J. J. Groenen and M. C. van Hemert from the University of Leiden have calculated the most likely configuration and have concluded that a boat-shaped geometry of the excited state is indeed the most stable (J. Am. chem. Soc. 112, 5447; 1990). There are many who would have put out flags at that stage, and turned their attention to the next problem on their agenda. But chemists are different. They want a picture of what has happened, and what may happen with other similar molecules.

So this interesting article concludes with an interpretation of what the Cray machine has said in almost anthropomorphic language. With seven electrons (one excited) in a planar conjugated system, the nitrogen atom would no longer be firmly bound to its adjacent carbons, with the consequence that the electron states on those two carbons "rehybridize themselves" so as to form bonds with the nitrogen atom more nearly like those in aliphatic carbon compounds. There follows entirely plausible brooding about the circumstances in which other molecules may behave similarly. Another rule of thumb seems to be in the making.

There is, of course, nothing wrong with such a way of doing business. Nobody plans to erect a generalization on the basis of a single calculation which, in the case of pyridine, has provided a neat confirmation of an experimental result and which only fortuitously provides grist to the mill, familiar enough to chemists, of rehybridization (which is but a name for the grouping of valence electrons on single atoms into new, but equivalent, linear combinations). But people with a more purist caste of mind would instead be worrying about questions such as whether the Born-Oppenheimer approximation that usually justifies the supposition that nuclei are immovable on the timescale of electron calculations can be valid for the excited state of pyridine. Perhaps it is not surprising that chemists lose patience with them.

John Maddox 